\title{
A Pilot Study to Compare Meal-Triggered Gastric Electrical Stimulation and Insulin Treatment in Chinese Obese Type 2 Diabetes
}

\author{
Simon Kin-Hung Wong, FRCSEd, Alice Pik-Shan Kong, MD, 2,3 Andrea On-Yan Luk, MRCP, \\ Risa Ozaki, FRCP, ${ }^{2}$ Vanessa Wan-Sze Ng, ${ }^{2}$ MRCP, Harold E. Lebovitz, MD, \\ Enders Kwok-wai Ng, MD, and Juliana Chung-Ngor Chan, MD ${ }^{2,3}$
}

\begin{abstract}
Background: Gastrointestinal electromodulation therapy is a novel alternative for achieving diabetes control without traditional bariatric surgery. We compared the efficacy of a meal-initiated implantable gastric contractility modulation (GCM) device with that of insulin therapy in obese Chinese type 2 diabetes (T2D) patients, for whom oral antidiabetes drugs (OADs) had failed.

Patients and Methods: Sixteen obese (body mass index, $27.5-40.0 \mathrm{~kg} / \mathrm{m}^{2}$ ) T2D patients with a glycated hemoglobin (HbA1c) level of $>7.5 \%$ on maximal doses of two or more OADs were offered either insulin therapy $(n=8)$ or laparoscopic implantation of a GCM $(n=8)$. We compared changes in body weight, waist circumference (WC), and HbA1c level 1 year after surgery.

Results: The GCM and insulin groups had similar baseline body weight and HbA1c. At 12 months, body weight $(-3.2 \pm 5.2 \mathrm{~kg}, P=0.043)$ and $\mathrm{WC}(-3.8 \pm 4.5 \mathrm{~cm}, P=0.021)$ fell in the GCM group but not in the insulin group $(P<0.05$ for between-group difference). At 6 and 12 months, the HbA1c level fell by $1.6 \pm 1.1 \%$ and $0.9 \pm 1.6 \%$ $(P=0.011)$, compared with $0.6 \pm 0.3 \%$ and $0.6 \pm 0.3 \%(P=0.08)$ for the insulin group $(P=0.15$ for between-group difference). The mean 24-h systolic blood pressure (BP) fell by $4.5 \pm 1.0 \mathrm{~mm} \mathrm{Hg}$ in the GCM group $(P=0.017)$ but not in the insulin group. The GCM group required fewer antidiabetes medications $(P<0.05)$ and BP-lowering drugs $(P<0.05)$ than the insulin group. A subgroup analysis showed that patients with a triglyceride level of $<1.7 \mathrm{mmol} / \mathrm{L}$ had a tendency toward a lower HbAlc level $(P=0.090)$ compared with the controls.

Conclusions: In obese T2D patients for whom OADs had failed, GCM implantation was a well-tolerated alternative to insulin therapy, with a low triglyceride level as a possible predictor for glycemic response.
\end{abstract}

\section{Introduction}

N 2011 THERE WERE 366 MILLION PEOPLE with type 2 diabetes (T2D), and the figure is expected to rise to 522 million by $2030 .{ }^{1}$ Obesity accounts for about $60 \%$ of the risk of developing diabetes, ${ }^{2}$ and more than $60 \%$ of T2D patients are obese. ${ }^{3}$ The metabolic disturbances in these patients, mediated by varying degrees of insulin resistance and impaired insulin secretion, are amplified by coexisting obesity. In 2010, a national survey in China reported the prevalence of prediabetes, obesity, and diabetes in the population to be $50 \%$, $50 \%$, and $11.6 \%$, respectively. ${ }^{4}$ Only $30 \%$ were receiving treatment, and among those, $40 \%$ had a glycated hemoglobin (HbA1c) level of $<7 \%$. Controlling hyperglycemia without

Departments of ${ }^{1}$ Surgery and ${ }^{2}$ Medicine and Therapeutics, Faculty of Medicine, The Chinese University of Hong Kong, The Prince of Wales Hospital, Hong Kong Special Administrative Region, China.

${ }^{3}$ Hong Kong Institute of Diabetes and Obesity, The Chinese University of Hong Kong, Hong Kong Special Administrative Region, China.

${ }^{4}$ Division of Endocrinology, The State University of New York Health Science Center at Brooklyn, Brooklyn, New York.

This study was presented as a poster at the $73^{\text {rd }}$ Scientific Sessions of the American Diabetes Association in June 2013 in Chicago, Illinois [Wong SK, Kong APS, Ozaki R, Ng V, Lebovitz HE, Ng EKW, Chan JC: A pilot prospective case-control study to compare the efficacy of laparoscopic placement of gastric contraction modulator (TANTALUS II ${ }^{\circledR}$ ) versus supplementary insulin treatment in obese type 2 diabetic patients].

This study is registered at ClinicalTrials.gov with clinical trial registration number NCT00975533. 
weight gain is a major unmet need in T2D due to the weightpromoting effects of many oral antidiabetes drugs (OADs), notably, sulfonylureas, thiazolidinedione, and insulin. ${ }^{5} \mathrm{Al}-$ though intensive lifestyle modification can cause weight loss with improved glycemic control, ${ }^{6,7}$ such intervention is laborintensive, and its long-term effects on diabetes-related morbidity, such as cardiovascular disease, are yet to be confirmed. ${ }^{8}$

Bariatric surgery, gastric electrical stimulation, and endoscopic duodenal sleeve placement have been shown to cause weight reduction and to improve diabetes control. ${ }^{9-11}$ The success of these operations has led to increasing recognition of the importance of dysregulation of the neuroendocrine function of the gastrointestinal tracts in modulating satiety and energy metabolism. In animal studies, nonexcitatory antral electrical stimulation increased the antral contraction amplitude and vagal afferent firing to the central nervous system. ${ }^{12}$ Chronic gastric electrical stimulation reduced food intake and induced weight loss in a canine model. ${ }^{13}$ In human studies, gastric electrical stimulation caused weight loss in obese individuals, although the sample size was small with variable effects. ${ }^{14}$

The DIAMOND (TANTALUS ${ }^{\mathrm{TM}}$ II) (MetaCure, KfarSaba, Israel) is a new gastric stimulatory device that uses gastric contraction modulation to enhance gastric antral contractility during food ingestion. ${ }^{15,16}$ In open-label controlled trials, the TANTALUS II improved glycemic control and caused weight loss in T2D patients without OADs. ${ }^{15,16}$ The device maintains the intrinsic rhythm of the stomach by sensing the native slow-wave activity and synchronizing the delivery of the gastric contraction modulator (GCM) stimulatory signal. The result is an increase in the force of the contractions while the stomach distends. Enhancement of stomach contractility with a GCM was shown to increase vagal afferent activity in animals. In open-label controlled trials, TANTALUS II improved glycemic control and caused weight loss in T2D patients uncontrolled with OADs. ${ }^{15,16}$ Despite these encouraging data, the efficacy of this device has not been directly compared with that of insulin therapy, a popular treatment option after OAD failure. In addition, the effectiveness of this device in the Chinese population has not been evaluated. In this pilot study, we compared the effect of GCM stimulation to insulin therapy in obese Chinese T2D patients for whom OADs and standard lifestyle modification were unsuccessful.

\section{Patients and Methods}

\section{Inclusion and exclusion criteria}

This was a single-center study conducted at a university teaching hospital in Hong Kong. Between January 2010 and May 2012, we used a diabetes registry to identify Chinese patients with T2D between 18 and 60 years of age with a disease duration of at least 2 years, a body mass index (BMI) of $27.5-40 \mathrm{~kg} / \mathrm{m}^{2}$, and $\mathrm{HbA} 1 \mathrm{c}$ level of $>7.5 \%$ treated with two or more OADs. We invited them for an interview at the Diabetes Centre for consideration of treatment intensification, including GCM implantation. Patients with an $\mathrm{HbA1c}$ level of $>10 \%$ or a fasting serum C-peptide level of $<0.5 \mu \mathrm{g} / \mathrm{L}$ were excluded and referred for initiation of insulin. Patients treated with thiazolidinedione or a dipeptidyl peptidase-4 inhibitor were allowed to participate if their body weight had been stable in the previous 6 months. However, patients taking a glucagon-like peptide-1 agonist were excluded from study. Patients with significant thyroid, renal, or hepatic diseases, advanced malignancies, active psychiatric illnesses, or substance abuse problems and those enrolled in weight loss programs or treated with anti-obesity drugs were excluded.

Upon recruitment, all of the patients were reviewed for optimization of the dosages of their OAD regimens, complication screening, reinforcement of medication compliance, and self-care, including self-monitoring of blood glucose (SMBG). Diabetic sensory neuropathy was accessed by clinical assessment and quantitative sensory testing of proprioception, vibration, and monofilament sensation, and autonomic neuropathy was assessed by reviewing symptoms of sexual dysfunction and gastroparesis. Patients were jointly interviewed by endocrinologists and surgeons, and the nature of GCM implantation as an alternative to insulin therapy for glycemic control was explained to them (study arm). Patients who refused GCM implantation were given insulin therapy (INS group). In the latter group, we selected subjects on a 1:1 basis, matched for age and HbAlc level, as the control arm for comparison with the GCM group. All of the patients gave written informed consent. The study was approved by the Chinese University of Hong Kong Clinical Research Ethics Committee.

\section{Intervention}

The implantation of the TANTALUS II system has been described previously. ${ }^{15}$ In brief, it consists of three pairs of bipolar electrodes and a rechargeable pulse generator, which are implanted with an external telemetry charger. The pulse has unique characteristics (nonexcitatory signal), which are set by a programmer during the initial installation of the device.

The bipolar electrodes were implanted by laparoscopic surgery, with one pair positioned in the fundus to detect food ingestion and the other two pairs positioned on the anterior and posterior antral areas to provide electrical stimulation to the antrum. The electrodes were connected to the pulse generator, which was implanted in a surgically created pocket in the anterior abdominal subcutaneous fat depot. Food ingestion was detected by the fundal electrode followed by activation of the pulse generator to provide an electrical pulse to the antral electrodes.

Following pre-assessment, the patients were admitted 1 day before implantation for preoperative preparation and optimization of glycemic control using insulin infusion, if necessary. Surgery was performed with the patient under general anesthesia. The patients were allowed to resume their normal diet the following morning and were discharged when they became fully ambulatory with satisfactory pain control.

\section{Treatment initiation and verification}

The GCM device was activated 1 week after the operation, when the pulse generator was programmed to deliver the appropriate signal triggered by the fundal electrode, which detected food intake. Food-mediated stimulation continued for $90 \mathrm{~min}$ after activation. After pre-assessment, the patients in the INS group were taught by the nurse educator and instructed to start insulin 1 week later, at a starting dose of $0.1-$ 0.2 units $/ \mathrm{kg} /$ day. The patients in both groups returned 4 weeks after treatment initiation to ensure that they were 
taking insulin or that the device was functioning. All of the patients had access to the research nurses via telephone, for early review as needed.

The patients in both intervention groups were asked to perform SMBG and measure their fasting blood glucose and predinner blood glucose at least twice weekly during the study. They were also requested to perform a four time-point blood glucose profile (fasting, prelunch, predinner, and bedtime) once during the week prior to the scheduled clinic visits.

After treatment initiation and verification at Week 4, all of the patients returned at 3, 6, 9, and 12 months for review of their clinical status and treatment by the nurses and endocrinologist fellows at the Diabetes Centre. For the INS group, dosages were individually titrated to avoid excessive weight gain and risk of hypoglycemia, taking into consideration patients' preferences. Both SMBG (aiming at a fasting blood glucose level of $<7 \mathrm{mmol} / \mathrm{L}$ and 2 -h postprandial blood glucose level of $<10 \mathrm{mmol} / \mathrm{L}$ ) and laboratory results (HbA1c and fasting plasma glucose) were used to individualize treatment. For the GCM group, the patients were reviewed by the programmer regarding adjustment of the device. Insulin was started as rescue therapy in patients with an HbA1c level of $\geq 9.0 \%$ after 3 months of GCM implantation. The insulin dosage was individualized as described for the INS group.

\section{Outcome measures}

All of the patients underwent a baseline assessment within a 4-week period before GCM implantation or insulin intervention. For the GCM group, the device was activated 1 week after the operation. All patients were subsequently reviewed at 3, 6, 9, and 12 months by evaluation of anthropometric indices, including body weight, BMI, waist circumference (WC), and body fat percentage as measured by bioimpedance (model TBF-300A body composition analyzer; Tanita Corp., Tokyo, Japan), with patients wearing light clothing. Fasting blood samples were collected to assess glycemic (HbA1c and fasting plasma glucose) and lipid (total cholesterol, triglyceride [TG], and high-density lipoprotein cholesterol) controls. All of the patients also undertook 24-h ambulatory blood pressure (BP) monitoring (Oscar $2^{\circledR}$; SunTech Medical, Morrisville, NC) before and 12 months after the intervention.

\section{Statistical analyses}

A statistical analysis was performed using IBM Statistical Package for Social Sciences Program version 21.0 software (SPSS, Chicago, IL). As this was a pilot study of GCM implantation without previous comparative data for the Chinese population, the sample size was not calculated. Nominal or categorical variables were presented as count with percentage per category and compared between groups using the $\chi^{2}$ test or Fisher's exact test, as appropriate. Continuous data were presented as mean and SEM. We used the MannWhitney $U$ test and the Wilcoxon signed-rank test for between-group comparisons for unpaired and paired data, respectively. The effects of different treatments on changes in body composition and metabolic control over time were compared using repeated-measures analysis of variance (rANOVA). The Greenhouse-Geisser and Huynh-Feldt estimates were used as correction factors according to the estimates $(\varepsilon)$ of sphericity. A value of $P<0.05$ was considered statistically significant.

\section{Results}

Between 2009 and 2012, 318 patients who fulfilled the inclusion criteria were identified and invited for interview. Of these, 294 patients were excluded because of commencement of insulin treatment prior to the interview $(n=115)$, use of submaximal dosages of OAD $(n=135)$, HbA1c level of $>10 \% \quad(n=10)$, advanced malignancy or significant comorbidities $(n=21)$, active psychiatric problems $(n=7)$, anticipated magnetic resonance imaging examination $(n=1)$, or refusal of insulin and surgical treatment $(n=10)$. Of the remaining 19 patients, eight patients agreed to GCM implantation, and the remaining 11 patients opted for insulin therapy.

In the GCM group, five men and three women (mean $\pm \mathrm{SE}$ age, $43.9 \pm 3.7$ years) underwent laparoscopic implantation of a GCM. Their mean body weight was $80.4 \mathrm{~kg}$, their BMI was $29.4 \mathrm{~kg} / \mathrm{m}^{2}$, and their disease duration was $14.2 \pm 2.0$ years. The fasting plasma glucose level was $10.8 \pm 0.9 \mathrm{mmol} / \mathrm{L}$, and the HbA1c level was $9.1 \pm 0.3 \%$. Of the 11 patients treated with insulin, we selected eight patients, matched for age, HbA1c, fasting C-peptide levels, and diabetes complication, as the control group (Table 1).

Table 1. Baseline Clinical and Biochemical

Characteristics of Obese Type 2 Diabetes

Patients Treated With Supplementary INSULIN OR IMPLANTED WITH a GASTRIC CONTRaCTILITy Modulator

\begin{tabular}{|c|c|c|c|}
\hline & $\begin{array}{l}\text { GCM } \\
\text { group } \\
(\mathrm{n}=8)\end{array}$ & $\begin{array}{c}\text { INS } \\
\text { group } \\
(\mathrm{n}=8)\end{array}$ & $\mathrm{P}$ value \\
\hline Male (\%) & $5(63 \%)$ & $6(75 \%)$ & 1.000 \\
\hline Age (years) & $43.9(3.7)$ & $49.3(2.6)$ & 0.382 \\
\hline Body weight (kg) & $80.4(4.0)$ & $86.0(4.3)$ & 0.279 \\
\hline $\begin{array}{l}\text { Body mass } \\
\text { index }\left(\mathrm{kg} / \mathrm{m}^{2}\right)\end{array}$ & $29.4(0.7)$ & 30.8 (1.6) & 0.202 \\
\hline $\begin{array}{l}\text { Waist } \\
\quad \text { circumference }(\mathrm{cm})\end{array}$ & $98.9(0.8)$ & $104.5(3.0)$ & 0.234 \\
\hline Body fat $(\%)$ & $33.5(2.4)$ & $28.6(1.5)$ & 0.105 \\
\hline $\begin{array}{l}\text { Duration of } \\
\text { diabetes (years) }\end{array}$ & $14.3(2.0)$ & $11.0(2.4)$ & 0.279 \\
\hline $\begin{array}{l}\text { Fasting C-peptide } \\
\qquad(\mu \mathrm{g} / \mathrm{L})\end{array}$ & $2.9(0.5)$ & $2.9(0.3)$ & 0.959 \\
\hline $\mathrm{HbA} 1 \mathrm{c}(\%)$ & $9.1(0.3)$ & $8.9(0.3)$ & 0.798 \\
\hline \multicolumn{4}{|l|}{ DM drug usage } \\
\hline Sulfonylureas & $7(88 \%)$ & $5(63 \%)$ & 0.569 \\
\hline Metformin & $8(100 \%)$ & $8(100 \%)$ & 1.000 \\
\hline Thiazolidinedione & $0(0 \%)$ & $2(25 \%)$ & 0.467 \\
\hline $\begin{array}{l}\alpha \text {-Glucosidase } \\
\text { inhibitors }\end{array}$ & $2(25 \%)$ & $3(37.5 \%)$ & 1.000 \\
\hline DPP-4 inhibitors & $0(0 \%)$ & $1(13 \%)$ & 1.000 \\
\hline \multicolumn{4}{|l|}{ DM complications } \\
\hline Retinopathy & $1(13 \%)$ & $2(25 \%)$ & 0.467 \\
\hline Neuropathy & $0(0 \%)$ & $0(0 \%)$ & 1.000 \\
\hline Cardiovascular & $1(13 \%)$ & $0(0 \%)$ & 1.000 \\
\hline Nephropathy & $0(0 \%)$ & $0(0 \%)$ & 1.000 \\
\hline
\end{tabular}

Data are mean (SEM) values or number (\%), as indicated.

DM, diabetes mellitus; DPP-4, dipeptidyl peptidase-4; GCM, gastric contractility modulator; HbAlc, glycated hemoglobin; INS, insulin. 


\section{Body composition}

During the 12-month study period, there were significant between-group differences for absolute weight loss $(P=0.027$, rANOVA) in favor of the GCM group (Fig. 1). In the GCM group, there was a significant reduction in the absolute (6 months, $-4.1 \pm 1.8 \mathrm{~kg} ; 12$ months, $-3.2 \pm 1.9 \mathrm{~kg} ; P=0.045$, rANOVA) and percentage change in body weight ( 6 months, $-5.3 \pm 2.1 \%$; 12 months, $-4.2 \pm 2.3 \% ; P=0.058$, rANOVA). The percentage excessive weight loss and the percentage excessive BMI loss at 12 months were $43.8 \pm 24.5 \%$ and $45.0 \pm 25.7 \%$, respectively. The INS group showed an increase in absolute body weight ( 6 months, $+1.0 \pm 0.9 \mathrm{~kg} ; 12$ months, $+1.4 \pm 1.1 \mathrm{~kg} ; P=0.289$, within-group rANOVA) and percentage change in body weight $(6$ months, $+0.7 \pm 1.1 \% ; 12$ months, $+1.2 \pm 1.3 \% ; P=0.558$, rANOVA). There were also significant between-group differences in the changes of WC $(P=0.016)$ and fat percentage $(P=0.055)$ in favor of the GCM group (Fig. 1).

\section{Glycemic control}

In the INS group, the mean starting dose of insulin was $8.3 \pm 0.6$ units/day at baseline, which was increased to $18.9 \pm 3.2$ units/day at 6 months and $28.0 \pm 6.8$ units/day at 12 months $(P=0.052)$. This was accompanied by a decrease in the HbA1c level from $8.9 \pm 0.3 \%$ to $8.3 \pm 0.5 \%$ at 6 months and to $8.4 \pm 0.5 \%$ at 12 months $(P=0.080$, rANOVA). In the GCM group, the HbA1c level fell from $9.1 \pm 0.3 \%$ to $7.5 \pm 0.3 \%$ at 6 months and to $8.2 \pm 0.5 \%$ at 12 months $(P=0.012$, rANOVA). No patients required rescue insulin at 6 months, and two patients $(25 \%)$ required it at 12 months. Although the HbA1c level in the GCM group fell significantly $(P=0.011)$ by $1.6 \pm 0.4 \%$ at 6 months, there was a rebound to $0.9 \pm 0.6 \%$ at 12 months with similar $\mathrm{HbA} 1 \mathrm{c}$ levels between the two groups at month 12 (GCM group vs. INS group, $8.2 \pm 0.5 \%$ vs. $8.4 \pm 0.5 \% ; P=0.959$ ) (Table 2 ), and no significant difference was observed in the HbA1c change between the two groups $(P=0.150$, rANOVA) (Fig. 1).
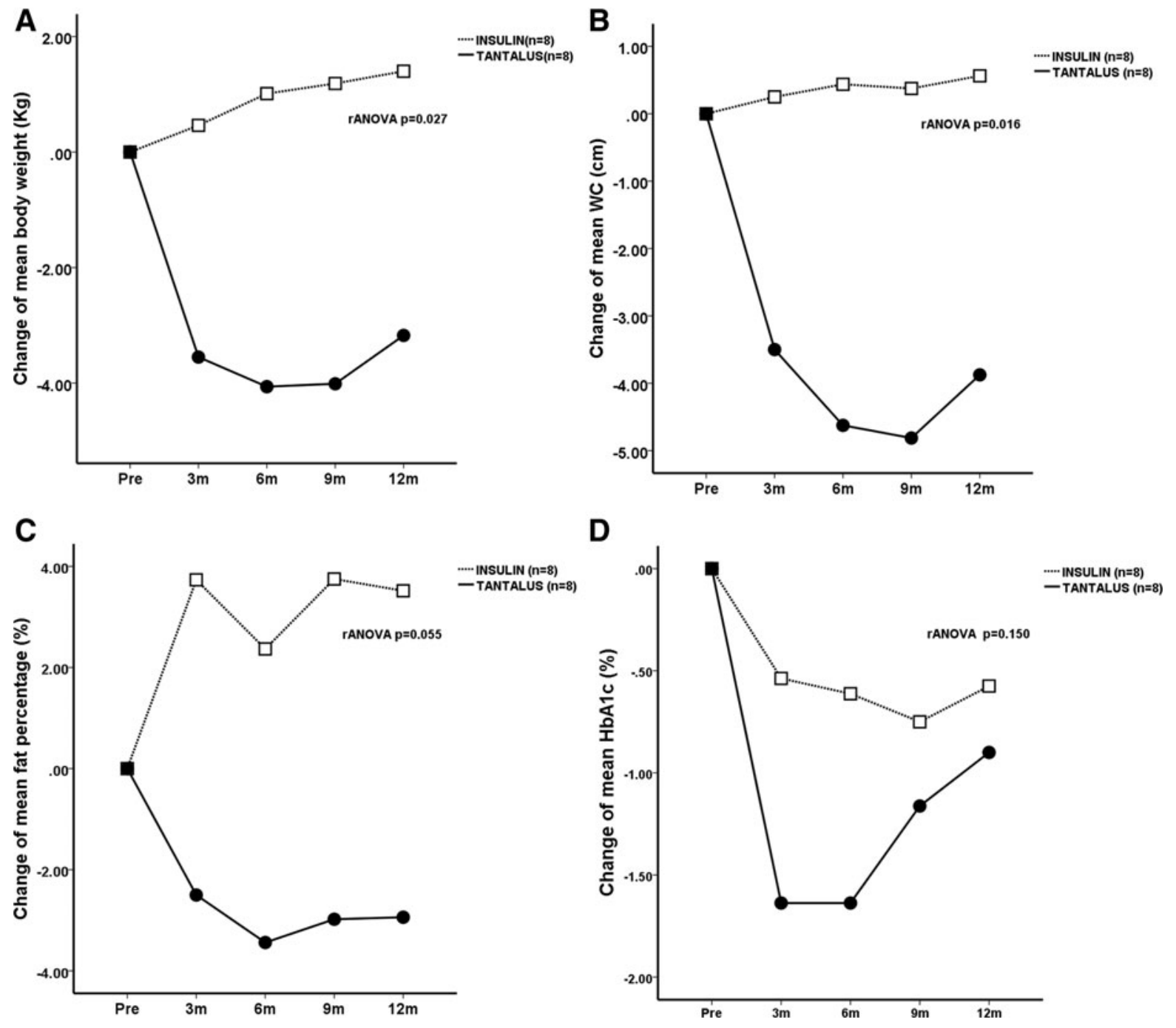

FIG. 1. Changes in body composition and glycated hemoglobin (HbA1c) in obese Chinese type 2 diabetes patients before and after treatment with supplementary insulin (open squares) or a gastric contractility modulator (TANTALUS II) (solid circles): (A) body weight, (B) waist circumference (WC), (C) body fat percentage, and (D) HbA1c. The $P$ value represents the estimated difference between the two groups by repeated-measures analysis of variance (rANOVA). 
Table 2. Changes in Glycemic, Lipid, and Blood Pressure Control in Obese Type 2 Diabetes Patients Before and After Treatment with a Gastric Contractility Modulator or Insulin

\begin{tabular}{lccc}
\hline Glycemic control & GCM group & INS group & P value \\
\hline FPG level (mmol/L) & & & \\
Before & $10.8(0.9)$ & $9.7(0.9)$ & 0.574 \\
6 months & $7.2(0.6)$ & $8.6(0.7)$ & 0.105 \\
12 months & $8.4(0.8)^{\mathrm{a}}$ & $8.7(0.1)$ & 0.798 \\
HbA1c level (\%) & & & \\
Before & $9.1(0.3)$ & $8.9(0.3)$ & 0.798 \\
6 months & $7.5(0.3)$ & $8.3(0.5)$ & 0.130 \\
12 months & $8.2(0.5)^{\mathrm{a}}$ & $8.4(0.5)$ & 0.959 \\
Number of antidiabetes drugs & & \\
Before & $2.0(0.2)$ & $2.4(0.2)$ & 0.279 \\
6 months & $1.9(0.3)$ & $3.0(0.2)$ & $0.007^{\mathrm{b}}$ \\
12 months & $1.9(0.4)$ & $3.5(0.3)^{\mathrm{a}}$ & $0.005^{\mathrm{b}}$ \\
INS dose (units/day) & & & \\
0 month & 0 & $8.3(0.6)$ & $<0.001^{\mathrm{b}}$ \\
6 months & 0 & $18.9(3.2)$ & $<0.001^{\mathrm{b}}$ \\
12 months & $6.0(2.0)^{\mathrm{a}}$ & $28.0(6.8)$ & 0.178
\end{tabular}

Lipid control

Triglycerides $(\mathrm{mmol} / \mathrm{L})$

$\begin{array}{llll}\text { Before } & 2.0(0.3) & 2.3(0.4) & 0.721 \\ 6 \text { months } & 1.4(0.2) & 1.9(0.4) & 0.574 \\ 12 \text { months } & 1.8(0.3)^{\mathrm{a}} & 1.7(0.4)^{\mathrm{a}} & 0.645\end{array}$

Total cholesterol (mmol/L)

$\begin{array}{llll}\text { Before } & 5.0(0.3) & 4.9(0.3) & 0.798 \\ 6 \text { months } & 4.7(0.3) & 4.3(0.3) & 0.328 \\ 12 \text { months } & 4.8(0.3) & 4.2(0.2) & 0.195\end{array}$

HDL-cholesterol (mmol/L)

$\begin{array}{llll}\text { Before } & 1.05(0.04) & 1.10(0.09) & 0.798 \\ 6 \text { months } & 1.11(0.09) & 1.08(0.10) & 1.000 \\ 12 \text { months } & 1.11(0.10) & 1.07(0.10) & 1.000\end{array}$

24-h ambulatory BP

24-h systolic BP (mm Hg)

$\begin{array}{llll}\text { Before } & 129.0(2.9) & 130.6(2.7) & 0.721 \\ \text { 12 months } & 124.5(3.8)^{\mathrm{c}} & 132.9(1.8) & 0.065 \\ \text { Systolic BP change } & -4.5(1.0) & +2.3(2.6) & 0.038^{\mathrm{b}} \\ \text {-h diastolic BP (mm Hg) } & & \\ \text { Before } & 78.8(1.5) & 78.8(1.5) & 0.721 \\ \text { 12 months } & 77.0(1.3) & 79.1(2.1) & 0.442 \\ \text { Diastolic BP change } & -1.9(0.8) & +0.4(1.3) & 0.105\end{array}$

Number of anti-HT drugs

$\begin{array}{llll}\text { Before } & 1.0(0.2) & 1.6(0.3) & 0.798 \\ 12 \text { months } & 0.8(0.2)^{\mathrm{c}} & 1.6(0.3) & 0.050^{\mathrm{b}}\end{array}$

Data are mean (SEM) values.

${ }^{a} P<0.05$ within groups was estimated by repeated-measures analysis of variance.

${ }^{\mathrm{b}} P$ value between groups was estimated by the Mann-Whitney $U$ test.

${ }^{\mathrm{c}} P<0.05$ within groups was estimated by the Wilcoxon signedrank test.

BP, blood pressure; FPG, fasting blood glucose; GCM, gastric contractility modulator; HbA1c, glycated hemoglobin; HDL, highdensity lipoprotein; HT, hypertension; INS, insulin.

There was a significant increase in the number of antidiabetes drugs used in the INS group $(P=0.018)$, with only two patients in the GCM group requiring additional insulin, and there were significant differences between the insulin dosages of the two groups at month 12 (Table 2).

\section{Lipid and BP control}

At 12 months, both groups had similar $(P=0.080$ for between-group difference) and significant reductions in TG levels, with no changes in serum total cholesterol and highdensity lipoprotein cholesterol levels (Table 2). The 24-h mean systolic BP was reduced from $129.0 \pm 2.9 \mathrm{~mm} \mathrm{Hg}$ at month 0 to $124.5 \pm 3.8 \mathrm{~mm} \mathrm{Hg}$ at month $12(P=0.017)$ in the GCM group, compared with from $130.6 \pm 2.7 \mathrm{~mm} \mathrm{Hg}$ to $132.9 \pm 1.8 \mathrm{~mm} \mathrm{Hg}$ $(P=0.325)$ for the INS group. The reduction in systolic BP in the GCM group $(-4.5 \pm 1.0 \mathrm{~mm} \mathrm{Hg})$, compared with an increase in the INS group $(+2.3 \pm 2.6 \mathrm{~mm} \mathrm{Hg})$, was significant $(P=0.038)$. The 24 -h mean diastolic BP did not change significantly in either group. At 12 months, the number of antihypertensive drugs was reduced by $0.8 \pm 0.2(P<0.05)$ in the GCM group and increased by $1.6 \pm 0.3(P=0.050)$ in the INS group $(P=0.05$ for between-group difference) (Table 2$)$.

\section{Adverse events}

There were no major adverse clinical events, and the GCM implantation was well tolerated. Three patients in the GCM group and one patient in the insulin group experienced symptomatic hypoglycemia during the study period, but none required assistance or hospitalization. All three patients from the GCM group developed hypoglycemia within the first 2 months after TANTALUS II implantation. None of these patients had a repeat hypoglycemia episode after discontinuation or reduction in the dosage of sulfonylureas. One patient in the INS group experienced hypoglycemia at 8 months with a basal insulin dosage of 8 units. This was subsequently reduced to 4 units.

\section{Hypertriglyceridemia and treatment responses}

At baseline, three (37\%) patients in the GCM group had high TG levels $(\geq 1.7 \mathrm{mmol} / \mathrm{L})$. Compared with the INS group, the low TG group had greater and more sustained weight loss, with a significant between-group difference in favor of the GCM group $(P=0.007$, between-group rANOVA). In the high TG group, weight changes were similar to those in the INS group ( $P=0.105$, between-group rANOVA) (Fig. 2). Indeed, two patients returned to their original weight after 12 months and required rescue insulin. The low TG group also had a tendency for the HbAlc level to be lower than that in the INS group $(P=0.090$, between-group rANOVA), with no between-group difference in the high TG group ( $P=0.438$, between-group rANOVA) (Fig. 2$)$.

\section{Discussion}

Insulin treatment is currently a standard therapy in patients who failed OADs, but weight gain after insulin therapy remained a challenge for most endocrinologists, especially in obese T2D patients. Gastrointestinal electrical stimulation has emerged as a new approach for weight loss and improving glycemic control. Data from animal models and preliminary data from human trials suggest that the gut-brain axis plays a role in the gastrointestinal electrical stimulation mechanism. To date, several different types of gastrointestinal electrical stimulation have been developed for weight reduction. The most widely known commercial ones are the DIAMOND (TANTALUS II) system, the Transcend ${ }^{\circledR}$ implantable gastric stimulator $\left(\mathrm{IGS}^{\circledR}\right)$ (Medtronic Transneuronix, Minneapolis, $\mathrm{MN}$ ), and $\mathrm{VBLOC}^{\circledR}$ vagal blocking therapy (EnteroMedics, 

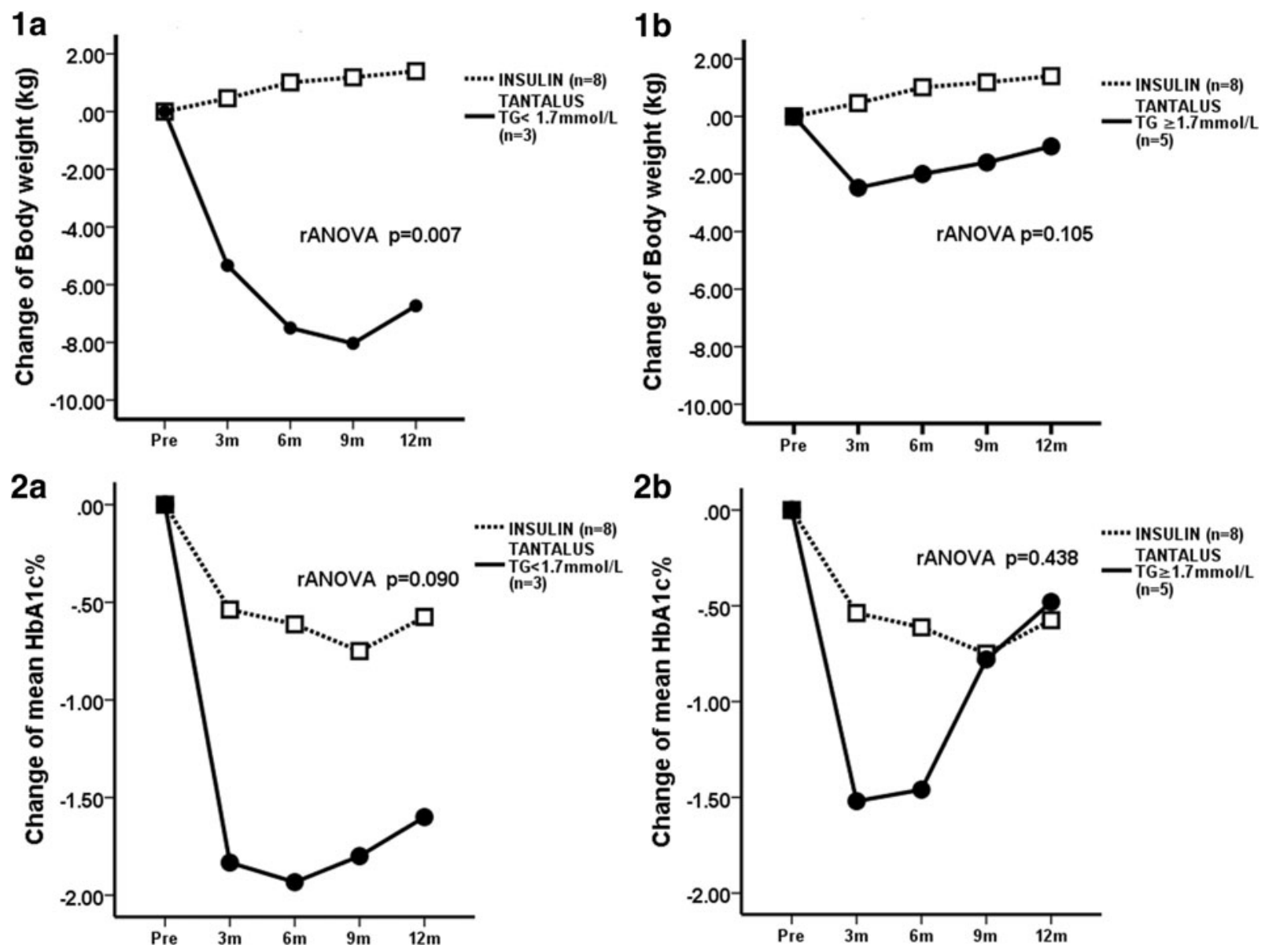

FIG. 2. Changes in body weight and glycated hemoglobin (HbA1c) in obese Chinese type 2 diabetes patients implanted with a gastric contractility modulator (TANTALUS II) (solid circles) stratified by baseline plasma triglyceride (TG), compared with the insulin treatment group (open squares): (1a and 1b) body weight and (2a and 2b) HbA1c between the insulin group and (1a and $\mathbf{2 a})$ the low TG TANTALUS group or (1) and $\mathbf{2 b})$ the high TG TANTALUS group. The $P$ value represents the estimated difference by repeated-measures analysis of variance (rANOVA).

St. Paul, MN). These devices have different target organs, different electrical stimulation patterns, and different locations for implantation of stimulation electrodes. Unlike VBLOC, DIAMOND (TANTALUS II) and Transcend IGS stimulate the stomach instead of the vagus nerve. The pattern of stimulation of DIAMOND (TANTALUS II) is also different from that of Transcend IGS, in which the train of short-wave stimulation in Transcend does not alter gastric contractility. Sample size for most of these studies was small, and there were no direct comparisons among these devices. However, almost all studies in each device group achieved statistically significant weight loss during the first 12 months, but weight loss over a longer follow-up period was rarely reported. Significant changes in reduction of Hb1Ac levels as well as BP were evident in most GCM studies ${ }^{15}$ and in one IGS study. ${ }^{17}$

In this study, we aimed to compare the effect of GCM in obese T2DM patients with suboptimal glycemic control with that of traditional insulin therapy. We included class I obesity (Asia-Pacific World Health Organization guideline definition of BMI as $>27.5 \mathrm{~kg} / \mathrm{m}^{2}$ ) as the degree of obesity in these patients does not fulfill current bariatric surgery criteria. However, we also like to exclude superobese subjects (BMI $>40 \mathrm{~kg} / \mathrm{m}^{2}$ ) as they are more suitable for aggressive weight loss by bariatric surgery. We also excluded patients on glu- cagon-like peptide-1 agonist therapy as we do not like to include another injection therapy such as glucagon-like peptide-1 agonist when we are comparing GCM with insulin injection therapy. Moreover, the incretin effect on appetite of glucagon-like peptide-1 agonist may also affect the outcome in the two study groups. We defined suboptimal glycemic control when the HbA1c level exceeded $7.5 \%$ as this cutoff point is commonly used to indicate of failure of first-line OAD treatment before initiation of insulin therapy. In this 12-month pilot study, obese Chinese T2D patients for whom OADs had failed and who had GCMs implanted had greater reductions in body weight, $\mathrm{WC}, \mathrm{HbA} 1 \mathrm{c}$, and 24-h mean BP than those treated with insulin. This was achieved using fewer medications for lowering blood glucose and BP. Although two of the eight patients in the GCM group required rescue insulin therapy, the dosage was considerably lower than that in the INS group (6 units vs. 28 units).

One important factor in diabetes management that needs to be addressed is the control of glycemia without weight gain. This therapeutic challenge is particularly difficult in obese T2D patients with long disease durations, who often require insulin treatment because of the failure of OADs. These obese patients often need high-dose insulin due to obesityassociated insulin resistance, which can lead to further weight 
gain and worsening of cardiometabolic risk factors, notably BP. ${ }^{18}$ Although bariatric surgical procedures, such as adjustable gastric banding, sleeve gastrectomy, or gastric bypass, were effective in improving glycemic control in T2D in randomized controlled trials, ${ }^{19-21}$ these operations were associated with significant, albeit small, operative morbidities. In insulin-requiring obese T2D patients who often have comorbidities, these invasive procedures can have a narrow risk-benefit ratio. For bariatric procedures with restrictive components, gastrointestinal symptoms and discomfort after feeding can further compromise the quality of life of these patients, who are already coping with multiple demands, such as lifestyle changes, injections, and SMBG. ${ }^{22,23}$

The results from this pilot study suggested that the delivery of nonexcitatory GCM signals using the TANTALUS II system was safe and well tolerated, with meaningful reductions in body weight, HbA1c, and BP in Chinese patients with T2D and moderate obesity. In these patients, the maximum weight loss of $5 \%$ occurred at 6 months, which was comparable to the experiences in white populations. ${ }^{14-16}$ The concomitant reduction in WC and body fat percentage supported the effects of GCM on body composition. By augmenting the food-stimulated gastric contraction, GCM has been shown to improve satiety and reduce food intake in both canine ${ }^{13}$ and human ${ }^{16}$ models.

In the Look AHEAD study, intensive lifestyle modification resulted in changes in body weight $(-8.6 \%)$, HbAlc $(-0.64 \%)$, and $\mathrm{BP}(-6.8 \mathrm{~mm} \mathrm{Hg})$ similar to those observed in the GCM group. ${ }^{6,7}$ However, these intensive lifestyle modification programs, which entailed weekly counseling sessions for 6 months and monthly reviews, were difficult to implement in non-clinical trial settings. To ensure that our results can be generalized to real-world practice, insulin-treated patients were managed in the usual manner with reinforcement of diabetes education and SMBG, along with insulin titration using an individualized approach. In light of the potential risk of severe hypoglycemia associated with intensive lowering of the blood glucose level ${ }^{24,25}$ and the fact that some of these patients already had cardiovascular and renal complications, the insulin dosage was increased gradually with additional oral drugs, to avoid excessive weight gain and hypoglycemia associated with high-dose insulin.

In this pilot study, the baseline HbA1c level was $9.1 \%$ and $8.9 \%$ in the GCM and INS groups, respectively. After 6 months, the insulin-treated group achieved a $0.6 \%$ reduction in HbA1c, although this was accompanied by weight gain and increased BP. In contrast, patients with a GCM implantation had a $1.6 \%$ reduction in $\mathrm{HbA} 1 \mathrm{c}$ at 6 months without insulin. In white T2D patients with a baseline $\mathrm{HbA} 1 \mathrm{c}$ of $8.4 \%$, the HbA1c level fell by $1.1 \% 6$ months after GCM implantation. ${ }^{15}$ In this study, the rapid fall in body weight and HbAlc level resulted in three of eight patients experiencing hypoglycemia, although none required assistance from a third person or hospitalization. Thus, it would be prudent to reduce the dosages of insulin or insulin secretagogues in patients with GCM implantation, to prevent hypoglycemia due to increased insulin sensitivity secondary to rapid weight loss.

After 6 months, glycemic control worsened in the GCM group, with two patients having returned to their original body weight and all of the patients requiring rescue insulin because of high HbA1c levels. Interindividual variation in treatment responses is one of the challenges of GCM therapy. Effect of GCM may be affected by underlying diabetic neuropathy, especially in our study group, when their mean duration of diabetes is 14.2 years. Some of our nonresponders may suffer from undiagnosed autonomic neuropathy and hence reduced the satiety response after stimulation. However, none of them reported clinical evidence of neuropathy during preoperative screening tests. On the other hand, Lebovitz et al. ${ }^{26}$ recently reported increased HbA1c responses to GCM therapy in patients with low TG and proposed that TG-associated lipotoxicity might interfere with gastric/neural-mediated pathways in the regulation of glycemic control in T2D. In support of these findings, we also found that patients with GCM implantation and a low TG level had a greater and more sustained reduction in body weight and HbA1c than those with high TG levels.

By 12 months, although the reduction in HbA1c level was similar between the INS and GCM groups, the difference in body weight and BP in favor of the GCM group was maintained. Of note is that the $5 \mathrm{~mm} \mathrm{Hg}$ reduction in systolic BP in the GCM group and $3 \mathrm{~mm} \mathrm{Hg}$ increase in the INS group, if maintained over time, are expected to translate into reduced numbers of cardiovascular events. ${ }^{27}$

\section{Limitations}

The major limitation of this study was the small sample size. In Hong Kong, the history of bariatric surgery is relatively limited, ${ }^{28}$ and most patients would not consider invasive therapy for obesity and/or diabetes. ${ }^{29}$ Our primary objective was to identify patients with clinical needs not met by conventional treatment. Although we were able to identify a large group of obese T2D patients who might benefit from GCM therapy, only $2.5 \%$ of patients who underwent screening and interview opted for GCM implantation. A randomized study design was preferred to avoid selection bias, although this would further increase the challenge of recruitment. In this study, we carefully matched the control group with the GCM group using age, gender, BMI, $\beta$-cell function as indicated by disease duration, and fasting $\mathrm{C}$ peptide level, although it remains plausible that our results might be confounded by unmeasured variables. That said, the modest effects on weight gain and BP following GCM therapy, despite the small sample size, are encouraging, calling for the use of a larger sample size for validation.

\section{Conclusions}

In this pilot study, we have demonstrated the safety, efficacy, and acceptability of the GCM therapy through implantation of the TANTALUS II device. Furthermore, in obese T2D patients for whom OADs had failed, GCM therapy was able to achieve a meaningful reduction in $\mathrm{HbA} 1 \mathrm{c}$ level with minimal use of insulin and OADs. It is important that, in contrast to the use of insulin, which tended to increase body weight and BP, GCM therapy was associated with a moderate reduction in these cardiovascular risk factors. The possibility of identifying patients with durable glycemic control for GCM treatment based on low TG will need to be verified using long-term and mechanistic studies.

\section{Acknowledgments}

We thank all of the patients at the Chinese University of Hong Kong-Prince of Wales Hospital Diabetes and Endocrine 
Centre and Diabetes Clinical Research Centre and the staff of these institutions for recruiting and managing them. The authors would also like to thank our research nurses, Mrs. Lindy Chan and Ms. Candice C.H. Lam, for their assistance in the data collection and coordination of this research project. This research is supported by a research grant from Metacure (USA) Ltd., Orangeburg, NY, awarded to the Chinese University of Hong Kong. The sponsor (MetaCure) had no involvement in the study design, collection, analysis, or interpretation of data, writing of the report, or the decision to submit the report for publication.

\section{Author Disclosure Statement}

H.E.L. is on the Scientific Advisory Board of MetaCure and has received honoraria. S.K.-H.W., A.P.-S.K., A.O.-Y.L., R.O., V.W.-S.N., E.K-w.N., and J.C.-N.C. declare no financial conflicts of interest exist.

\section{References}

1. Whiting DR, Guariguata L, Weil C, et al.: IDF Diabetes Atlas: global estimates of the prevalence of diabetes for 2011 and 2030. Diabetes Res Clin Pract 2011;94:311-321.

2. International Association for the Study of Obesity. www.iaso .org/policy/aboutobesity/ (accessed March 3, 2013).

3. Astrup A, Finer N: Redefining type 2 diabetes: 'diabesity' or obesity dependent diabetes mellitus. Obes Rev 2000;1:57-59.

4. $\mathrm{Xu} \mathrm{Y,} \mathrm{Wang} \mathrm{L,} \mathrm{He} \mathrm{J,} \mathrm{et} \mathrm{al.:} \mathrm{Prevalence} \mathrm{and} \mathrm{control} \mathrm{of}$ diabetes in Chinese adults. JAMA 2013;310:948-959.

5. Bailey CJ: The challenge of managing coexistent type 2 diabetes and obesity. BMJ 2011;342:d1996.

6. Look AHEAD Research Group: Reduction in weight and cardiovascular disease risk factors in individuals with type 2 diabetes - one-year results of the Look AHEAD trial. Diabetes Care 2007;30:1374-1383.

7. Look AHEAD Research Group; Wing RR: Long-term effects of a lifestyle intervention on weight and cardiovascular risk factors in individuals with type 2 diabetes mellitus: four year results of the Look AHEAD trial. Arch Intern Med 2010;170:1566-1575.

8. Look AHEAD Research Group: Cardiovascular effect of intensive lifestyle intervention in type 2 diabetes. $\mathrm{N}$ Engl $\mathrm{J}$ Med 2013;369:145-154.

9. Rubino F, Schauer PR, Kaplan LM, et al.: Metabolic surgery to treat type 2 diabetes: clinical outcomes and mechanisms of action. Annu Rev Med 2010;61:393-411.

10. Sanmiguel CP, Conklin JL, Cunneen SA, et al.: Gastric electrical stimulation with the TANTALUS ${ }^{\circledR}$ system in obese type 2 diabetic patients: effect on weight and glycemic control. J Diabetes Sci Technol 2009;3:964-970.

11. Rodriguez L, Reyes E, Fagalde P, et al.: Pilot clinical study of an endoscopic, removable duodenal-jejunal bypass liner for the treatment of type 2 diabetes. Diabetes Technol Ther 2009;11:725-732.

12. Peles S, Petersen J, Aviv R, et al.: Enhancement of antral contractions and vagal afferent signaling with synchronized electrical stimulation. Am J Physiol Gastrointest Liver Physiol 2003;285:G577-G585.

13. Eagon JC, Kelly KA: Effect of electrical stimulation on gastric electrical activity and emptying. Neurogastroenterol Motil 1995;7:39-45.

14. Greenway F, Zheng J: Electrical stimulation as treatment for obesity and diabetes. J Diabetes Sci Technol 2007;1:251-259.
15. Policker S, Haddad W, Yaniv I: Treatment of type 2 diabetes using meal-triggered gastric electrical stimulation. Isr Med Assoc J 2009;11:206-208.

16. Bohdjalian A, Ludvik B, Guerci B, et al.: Improvement in glycemic control by gastric electrical stimulation (TANTALUS $^{\mathrm{TM}}$ ) in overweight subjects with type 2 diabetes. Surg Endosc 2009;23:1955-1960.

17. Genev NM, Lau IT, Willey KA, et al.: Does insulin therapy have a hypertensive effect in type 2 diabetes? J Cardiovasc Pharmacol 1998;32:39-41.

18. Cigaina V: Long-term follow-up of gastric stimulation for obesity: the Mestre 8-year experience. Obes Surg 2004;14(Suppl 1):S14-S22.

19. Dixon JB, O’Brien PE, Playfair J, et al.: Adjustable gastric banding and conventional therapy for type 2 diabetes: a randomized controlled trial. JAMA 2008;299:316-323.

20. Ikramuddin S, Korner J, Lee WJ, et al.: Roux-en-Y gastric bypass vs intensive medical management for the control of type 2 diabetes, hypertension, and hyperlipidemia: the Diabetes Surgery Study randomized clinical trial. JAMA 2013;309:2240-2249.

21. Schauer PR, Kashyap SR, Wolski K, et al.: Bariatric surgery versus intensive medical therapy in obese patients with diabetes. N Engl J Med 2012;366:1567-1576.

22. Lee WJ, Wang W, Yu PJ, et al.: Gastrointestinal quality of life following laparoscopic adjustable gastric banding in Asia. Obes Surg 2006;16:586-591.

23. Lee WJ, Huang MT, Yu PJ, et al.: Laparoscopic vertical banded gastroplasty and laparoscopic gastric bypass: a comparison. Obes Surg 2004;14:626-634.

24. Leese GP, Wang J, Broomhall J, et al.: Frequency of severe hypoglycemia requiring emergency treatment in type 1 and type 2 diabetes: a population-based study of health service resource use. Diabetes Care 2003;26:1176-1180.

25. Dluhy RG, McMahon GT: Intensive glycemic control in the ACCORD and ADVANCE trials. N Engl J Med 2008; 358:2630-2633.

26. Lebovitz HE, Ludvik B, Yaniv I, et al.: Fasting plasma triglycerides predict the glycaemic response to treatment of type 2 diabetes by gastric electrical stimulation. A novel lipotoxicity paradigm. Diabet Med 2013;30:687-693.

27. Stratton IM, Cull CA, Adler AI, et al.: Additive effects of glycaemia and blood pressure exposure on risk of complications in type 2 diabetes: a prospective observational study (UKPDS 75). Diabetologia 2006;49:1761-1769.

28. Wong SK, Kong AP, So WY, et al.: Use of laparoscopic sleeve gastrectomy and adjustable gastric banding for suboptimally controlled diabetes in Hong Kong. Diabetes Obes Metab 2012;14:372-374.

29. Wong SK, Kong AK, Mui WL, et al.: Laparoscopic bariatric surgery: a five-year review. Hong Kong Med J 2009; 15:31-38.

Address correspondence to:

Enders Kwok-wai Ng, MD

Department of Surgery

4/F, Lui Che Woo Clinical Sciences Building

Prince of Wales Hospital 30-32, Ngan Shing Street

Shatin, New Territories, Hong Kong Special Administrative Region, China

E-mail: endersng@cuhk.edu.hk 